\title{
Low Profile Visualized Intraluminal Support (LVIS) Stent in Endovascular Coil Embolization of Cerebral Aneurysms: A Review
}

\author{
Ujjwol KC*, Gang Deng \\ Department of Interventional Radiology, Zhongda Hospital, Southeast University, Nanjing, China \\ Email: *ujjwolkhatrichhetri@hotmail.com
}

How to cite this paper: KC, U. and Deng, G. (2019) Low Profile Visualized Intraluminal Support (LVIS) Stent in Endovascular Coil Embolization of Cerebral Aneurysms: A Review. Open Journal of Radiology, 9, 93-104.

https://doi.org/10.4236/ojrad.2019.92009

Received: March 16, 2019

Accepted: April 7, 2019

Published: April 10, 2019

Copyright (อ 2019 by author(s) and Scientific Research Publishing Inc. This work is licensed under the Creative Commons Attribution International License (CC BY 4.0).

http://creativecommons.org/licenses/by/4.0/

\begin{abstract}
Cerebral or intracranial aneurysm is a leading cause of subarachnoid hemorrhage. It was initially treated with open surgical clipping but as rapid development of technology a less invasive endovascular coiling technique of aneurysm revolutionized the treatment. Due to tortuous anatomy of parent artery and complicated morphology and location of aneurysm there is ongoing challenge in the complete obliteration of aneurysms. To aid in the advances of treating aneurysm stent assisted endovascular coiling was introduced to give more scaffold support to parent artery and decrease events of coil protrusion from aneurysms. Many types and generation of stents were developed. One of the most recently introduced stent is low profile visualized intraluminal support (LVIS) stent. Due to its low-profile nature it can be used in 0.017-inch inner diameter microcatheter and reach small and complex vessels providing high aneurysmal neck coverage which was not possible through other traditional stent. In addition, its braided design with tantalum strands and radiopaque markers make it more visible during stent placement and post procedure stent evaluation. Despite of many advantages of LVIS stent it is related to high rate of thromboembolic complications and technical complications. Aim of this review paper was to evaluate therapeutic safety, effectiveness and feasibility of LVIS stent in endovascular coil embolization of intracranial aneurysms.
\end{abstract}

\section{Keywords}

Low Profile Visualized Intraluminal Support (LVIS),

Endovascular Treatment, Intracranial Aneurysms

\section{Introduction}

Cerebral aneurysm or intracranial aneurysm, is a cerebrovascular disorder caused 
by weakness in the inner muscular layer wall of cerebral artery or vein resulting in focal dilation or ballooning of the blood vessel. The dilated vessels can become thin and rupture without any threatening signs resulting in subarachnoid hemorrhage which can lead to stroke, coma or death. Most evident division of cerebral aneurysm is considering the ruptured and unruptured lesions. Cerebral aneurysms can be classified in terms of size (small $(<10 \mathrm{~mm})$, large $(10-25 \mathrm{~mm})$, and giant (>25 mm) in diameter) [1], location (Anterior circulation $90 \%$ and posterior circulation 10\%) [2]. Non-saccular aneurysms (fusiform, dolichoectatic, and dissecting aneurysms) are rare with less than $0.1 \%$ incidence [3]. Saccular or berry aneurysms is the most common form of cerebral aneurysm occurring in about $1 \%$ - $2 \%$ of the population and related to $80 \%-85 \%$ of non-traumatic subarachnoid hemorrhages [4] [5]. According to findings from an analysis of 68 prevalence studies data from 83 study populations prevalence of intracranial saccular aneurysms of $3.2 \%$ is with male: female ratio of 1:2 [6]. Intracranial aneurysms are seldomly acquired lesions; however, a rare heredity form has been related with conditions like Marfan's syndrome, Autosomal dominant polycystic kidney disease, Ehlers-Danlos syndrome type IV, fibromuscular dysplasia, sickle cell anemia, Moyamoya disease and brain arteriovenous malformation. About 5\% - 40\% of patients with autosomal dominant polycystic kidney disease have intracranial aneurysms, and $10 \%-30 \%$ of patients have multiple aneurysms [7]. An important risk factor of aneurysm is family history. Reversible risk factors like alcohol consumption, smoking and hypertension increase occurrence of aneurysm [8] [9].

\section{Background}

Endovascular techniques for treating aneurysms started back to the 1970s by a Russian neurosurgeon, MD Fjodor A. Serbinenko with the introduction of proximal balloon occlusion. During 1980s, there was high rate of procedural rupture and complications those treated with endovascular balloon occlusion technique [10]. Guido Guglielmi, MD, an American neuroradiologist invented the platinum detachable micro coil, which was used to treat the first human being in 1991 and their FDA approval in 1995 gave revolution to endovascular treatment of cerebral aneurysms [11].

Endovascular coiling has been regarded as a safe and effective alternative to surgical clipping of aneurysms [12] [13] [14]. The goal of both surgical clipping and endovascular coiling is to stop blood flow into the aneurysm. On the basis of aneurysm obliteration rate and without any evidence of aneurysmal recanalization or reoccurrence, treatment effectiveness is measured. Endovascular coiling is a good option for treating aneurysms because it is minimally invasive technique, which means no need to open skull, can be done in short time which ultimately lower anesthesia time. However, the important different between endovascular coiling and surgical clipping, includes how the aneurysm was closed. Because comparing both coiling does not physically approximate the inner blood vessel lining, blood flow to aneurysm may occur through the incomplete compac- 
tion of the coils. Nevertheless, due to the risk of coil herniation or migration responsible for thromboembolic complications coil embolization of complex-shaped and wide necked aneurysms remains challenging. Advanced techniques like balloon remodeling and stent-assisted coiling for endovascular treatment of complex and wide-necked aneurysms has been developed which allow compact coil packing hence preventing coil protruding into the parent artery [15]. In addition, stents may induce flow diversion effect hence reducing blood flow towards aneurysm and provides a scaffold support around aneurysm neck which helps in new endothelial tissue growth resulting vessel wall healing and preventing circulation towards aneurysms [16]. A new self-expandable stent, the Low-profile Visualized Intraluminal Support (LVIS) device is introduced for stent assisted coil embolization cerebral aneurysms.

\section{LVIS Device Description and Endovascular Procedure [17] [18]}

The LVIS stent (MicroVention Terumo, Tustin, California, USA), which offers an option between conventional coil assist stents and flow diverters, it was designed to improve the long-term efficacy of endovascular treatment while excluding impact on side branches. The LVIS is a self-expanding, hybrid closed cell, braided stent with nitinol wires and flared ends with proximal and distal radiopaque markers, and double helical tantalum strands to help in full visualization. Due to its small cell size $(<0.9 \mathrm{~mm})$ it can provide better parent artery protection against small sized coils around the aneurysm neck. It has high metal to surface coverage (23\% on average) as compared to other available stents which helps improving flow diversion.

The 2 different kinds of the stent: LVIS and LVIS Junior. The LVIS stent, which is suitable with a 0.021 -inch microcatheter is recommended for a parent artery diameter of $2.0-5.0 \mathrm{~mm}$ has a cell size of $1.0 \mathrm{~mm}$. It has four radiopaque tantalum markers on the proximal/distal ends with two radiopaque helical strands visible within body of the stent. The LVIS Junior stent, which is suitable with a 0.017 -inch microcatheter, has a cell size of $1.5 \mathrm{~mm}$ and is intended for vessels sized $2.0-3.0 \mathrm{~mm}$. It has three radiopaque tantalum markers at the proxim$\mathrm{al} /$ distal ends and 3 radiopaque helical strands visible throughout the body.

To deliver stent to the target site a $6 \mathrm{~F}-8 \mathrm{~F}$ guiding microcatheter is introduced through a femoral sheath into the carotid artery. Radiologic examination of target artery is performed using a biplane angiographic system, rotational angiography followed by three-dimensional reconstruction is performed to assess the aneurysm and parent artery morphology for accurate measurement. The LVIS device is available in 3 sizes $(3.5,4.5$, and $5.5 \mathrm{~mm}$ in diameter) with various lengths. Determination the stent size selection is done according to target parent artery diameter. Different stenting strategies can be done according to morphology, types or location of aneurysm some are as follows: 1) Semi jailing technique; 2) Bailout stent placement [18]; 3) Overlapping stenting (Y, X-stenting) 
[19]; 4) Bulging technique [20]; 5) Balloon remodeling followed by stenting [21].

\subsection{Anticoagulation and Antiplatelet Treatment [18]}

All patients prior to endovascular coiling procedure are heparinized after catheter is placed to prevent blood clotting. Blood coagulation factors are routinely monitored pre and post procedure. Patient with unruptured aneurysms are pretreated with Dual antiplatelet drugs (Aspirin $100 \mathrm{mg}$ and Clopidogrel $75 \mathrm{mg}$ ) to decrease platelet aggregation and inhibit thrombus formation for prevention of any primary and secondary thrombotic events. Loading dose of Dual antiplatelet drugs $300 \mathrm{mg}$ each should be given before stenting procedure for patient with acute ruptured aneurysmal subarachnoid hemorrhage to reduced risk of clinical vasospasm and delayed cerebral ischemia [22]. Post procedure patient are continued with daily maintenance dose of Aspirin and clopidogrel for some weeks as per patient need and followed by aspirin alone for lifelong.

\subsection{Clinical and Imaging Follow-Up}

Patient are clinically assessed according to Modified Rankin scale (mRS) [23] for any neurological disability or dependency in daily activities on the day of procedure and in follow up in 3 - 6 - 12 months (Table 1).

Various Imaging technique as Magnetic resonance angiography, Digital subtraction angiography are performed post procedure and in follow-up to evaluate aneurysmal occlusion, stent patency, stent position, stent migration, instent stenosis, aneurysmal reoccurrence or obliteration and any thromboembolic events. Angiographic results for grading of aneurysmal occlusion are assessed according to Modified Raymond-Roy Classification [24] (class I: complete obliteration, class II: residual neck, class IIIa: contrast opacification within the coil interstices of a residual aneurysm, class IIIb: contrast opacification outside the coil interstices, along the residual aneurysm wall).

\section{Review of Articles}

Regarding overall safety, effectiveness, feasibility and complications of LVIS

Table 1. Modified rankin scale.

\begin{tabular}{lc}
\hline \multicolumn{1}{c}{ Symptoms } & Grade \\
\hline No symptoms at all & 0 \\
No significant disability despite symptoms; able to carry out all usual duties and activities & 1 \\
$\begin{array}{l}\text { Slight disability; unable to carry out all previous activities, but able to look after own affairs } \\
\text { without assistance }\end{array}$ & 2 \\
$\begin{array}{l}\text { Moderate disability; requiring some help, but able to walk without assistance } \\
\text { Moderately severe disability; unable to walk without assistance, unable to attend to needs } \\
\text { without assistance }\end{array}$ & 3 \\
Severe disability; bedridden, incontinent, and requiring constant nursing care and attention & 5 \\
Dead & 6 \\
\hline
\end{tabular}


stent we reviewed various original articles related to LVIS Stent from 2012-2018. PUBMED, SCOPUS, GOOGLE SCHOLAR and RESEARCH GATE search engines were used using key words low profile visualized intraluminal support (LVIS) for articles search. We mainly focused on articles in english language about study related safety and effectiveness of LVIS, articles directly comparing LVIS stent with other stent in the endovascular treatment of cerebral aneurysms. Articles related to surgical clipping, endovascular coiling without stent assistance, animal study and non-English language were kept on exclusion criteria (Figure 1).

\subsection{Wei Su, Yisen Zhang et al. [25]}

In this single-center retrospective study they enrolled 218 patients with various types of intracranial aneurysms treated with LVIS stent. After the implantation of stent post procedural angiographic results of aneurysmal occlusion were evaluated as Raymond class 1 (139, 63.8\%), Raymond class 2 (75, 34.4\%), Raymond class $3(4,1.8 \%)$. All patients were clinically evaluated by $\mathrm{mRS}$ score after the procedure and at follow-up from 1 to 23 months. mRS score at follow-up were: 0 for $141(88.1 \%)$ patients, 1 for $15(9.4 \%)$ patients, 2 for $0(0.0 \%)$ patients, 3 for 1 $(0.6 \%)$ patients, 4 for $1(0.6 \%)$ patients, 5 for $0(0.0 \%)$ patients, and 6 for 2 (1.3\%) patients. 2 of 1 patient died cause of distal vessel perforation due to tip wire of LVIS stent and other patients died due to hemorrhage from recurrence of vertebral dissecting aneurysm leading to brain stem failure. According to this

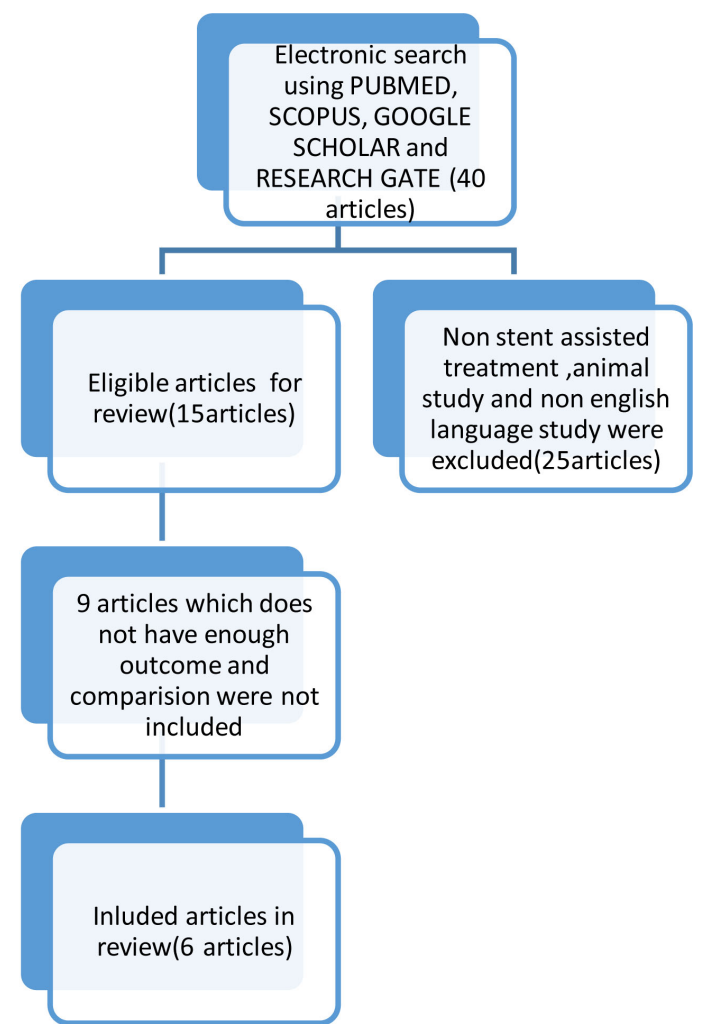

Figure 1. A flow diagram reflecting the literature review process. 
study LVIS stent deployment success rate was high (99.1\%) as compared to other laser cut stent studies because of high aneurysmal occlusion and low complication rate. However, this study concluded that to evaluate efficacy of LVIS in treatment of intracranial aneurysm larger patient population and longer follow up is needed.

\subsection{David Fiorella, Alan Boulos et al. [26]}

This study was prospective, multicenter, interventional study done in 21 UNITED STATE hospitals to assess the safety and effectiveness of LVIS stent in the coil embolization of wide necked intracranial aneurysms. Total of 153 patients were enrolled from the date July 2013 to October 2014. As per study protocol success rate asses by successful and stable stent placement with coverage of aneurysm neck and parent artery patency. Safety was evaluated as rate of stroke or death within 30 days to 1-year time interval. Effectiveness of treatment was assessed according to complete angiographic aneurysmal rate without any retreatment and stenosis ( $\geq 50 \%)$ of parent artery at 1-year follow-up. Success rate was 149 of 153 (97.3\%) patients. As per safety protocol total 14 (9.2\%) out of 153 patients had stroke (ischemic and hemorrhagic) events and 3 patients died followed by major stokes and additional 3 patients died of non-neurological cause (suicide, cardiac events and drug overdose) within time interval 30 days-1 year. Overall mortality was 6 (3.9\%) out of 153 patients. 14 patients did not follow up for 1-year angiographic assessment so out of 139 patients 128 (92.1\%) patients showed $\geq 95 \%$ aneurysmal occlusion and 132 (95\%) showed $\geq 90 \%$ occlusion. 6 patients (less than 5\%) required retreatment and no patients showed parent artery stenosis. Due to high success rate of stent deployment, high level of procedural safety and high occlusion rate of aneurysms US LVIS pivotal trial concluded LVIS stent as safe and effective tool in the treatment of wide necked cerebral aneurysms.

\subsection{Young Dae Cho \& Chul-Ho Sohn et al. [27]}

Total of 55 patients with saccular aneurysm treated with LVIS (LVIS, LVIS Jr) stent between October 2012 and February 2013 were included in this prospective, multicenter study done in 2 hospitals from south korea.27 patient treated with standard LVIS and 28 with LVIS Jr. This study was based in the midterm follow up (6 month) clinical and angiographic results in which follow-up imaging of 54 patients was done. According to study result 45 (81.8\%) aneurysm were successfully occluded with procedure related morbidity 2 (3.6\%) out of 55 patients. Midterm results show confirmation of 50 (92.6\%) complete aneurysmal occlusion, 3 (5.5\%) neck remanant, 1 (1.9\%) residual sac. This study pointed that LVIS was feasible in stent assisted endovascular coiling and can be used in the treatment of unruptured intracranial aneurysms but due high rate of instent stenosis 26 (86.7\%) out 30 aneurysms follow up angiographic result and segmentally incomplete stent expansion in 5 cases they concluded that further long 
term follow up monitoring is needed.

\subsection{Bradley a Gross, William J Ares et al. [28]}

This study was held between November 2013 and April 2018, total 64 patients were included in which 27 patients were treated with LVIS Jr and 37 patients were treated with ATLAS stent in endovascular coil embolization of cerebral aneurysms. Objective of this study was focused on clinical comparison of LVIS Jr and ATLAS on the basis of technical success and complication rate. According to their study results initial Raymond 1 occlusion rate of aneurysm of ATLAS was higher ( $57 \%$ vs $41 \%$ LVIS, $\mathrm{P}=0.03$ ), significant greater rate Raymond 1 or 2 aneurysmal occlusion in follow up angiography results (ATLAS 100\% vs 81\% LVIS, $\mathrm{P}=0.04$ ) with minimum rate of in-stent stenosis for ATLAS (ATLAS $0 \%$ vs $19 \%$ LVIS, $P=0.04$ ). As compared to those treated with LVIS stent this study demonstrates ALTAS stent has higher aneurysmal obliteration rate and lower in-stent stenosis rate.

\subsection{Huijian Ge, Xianli et al. [29]}

This single center, retrospective study was done in November 2014 to December 2015. Total of 190 patients with 208 intracranial aneurysms were undergone treatment with LVIS (92 patients with 96 aneurysms) and Enterprise stent (98 patients with 112 aneurysms). Aim of this study was to analyze the post procedure angiographic results, complication related to procedure (hemorrhagic and thromboembolic events) and clinical outcomes to compare between LVIS and Enterprise stent. Procedure related complication was $10.9 \%$ of patients and Enterprise $16.3 \%$. Thromboembolic events (8 LVIS, 14 Enterprise) $\mathrm{P}=0.263$ and hemorrhagic events (2 LVIS, 1 Enterprise) $\mathrm{P}=0.611$ with good clinical outcomes $(\mathrm{mRS}$ or $0-2)(\mathrm{P}=0.379)$ shows no statically significant difference between two stents. However, in case of initial complete or near complete occlusion of aneurysms LVIS (96.9\%) and Enterprise (88.4\%), LVIS shows higher rate of complete or near complete obliteration rate.

\subsection{Su-Yeon Park, Jae-Sang Oh et al. [30]}

This systematic literature review was done whether the low profile self-expandable stent (LVIS Jr and LEO baby) have higher incidence of thromboembolic complications or not as compared to another existing stent. In addition, they have done meta-analysis LVIS Jr vs LEO Baby stent on the basis of three outcomes (obliteration of aneurysm prior to 6 month follow-up and procedural and technical complications). To show the safety and efficacy of low profile self-expandable stents 11 suitable reports were taken for this systematic review. Total of 217 aneurysms were reported which were treated using various stenting techniques. Raymond class 1 and 2 obliteration was found in $87 \%$ of aneurysm on immediate post procedure angiographic results. Raymond class 1 and 2 was found in $79 \%$ and Raymond class 3 obliteration was found in $3 \%$ of treated aneurysms on 
3 - 6-month angiographic follow-up results. LVIS JR and LEO Baby shows low Reoccurrence rate $6.5 \%$ (1.3\% in LVIS, 5.7\% in LEO Baby) in midterm follow up results as compared to other available stents. Low profile self-expandable stents can be used in small parent artery and have more metal coverage due to which it can be useful and safe to treat intracranial aneurysms but have high post procedure thromboembolism rate $6.5 \%$ and technical complications. Meta-analysis concluded that there was no significant difference $(\mathrm{P}$ value $=0.953)$ between LVIS Jr and LEO Baby stent in obliteration rate at 6 months angiographic follow up but periprocedural complication rate was significantly lower $(\mathrm{P}=0.008)$ in LVIS Jr as compared to LEO Baby.

\section{Summary}

In 1997 Higasida first published study about stenting technique in endovascular coiling of ruptured intracranial aneurysms; since then there is rapid development in the field of stent assisted coil embolization of cerebral aneurysms [31]. Purpose of endovascular technique is complete obliteration, stopping intracranial blood supply to aneurysms hence preventing recanalization for the protection against aneurysmal rupture. However small, wide necked and other complex anatomy cerebral aneurysm are still challenging for the treatment [32] [33]. Due to rapid growth in technology many newer generation self-expandable stents are already developed; one of them is Low-profile Visualized Intraluminal Support device (LVIS and LVIS Jr). LVIS offers advantages over other available stent like its low-profile nature, more visibility [34] (3 radiopaque nitinol strands visible through the body as well as three proximal and distal radiopaque markers), and closed cell with braided design which ultimately increases metal to vessel neck surface area resulting in better flow diversion effect [32] [35].

According to study [26] [34] [36] LVIS stent was highly effective in the treatment of small, wide-necked aneurysm. In previous report LVIS VS Enterprise stent they compared both stent; as a result there was no significant difference in clinical outcomes and complication of both stent but LVIS stent resulted in high complete or near complete occlusion rate of aneurysms [29]. On the basis of hemodynamic effect of stent to reduce blood flow to aneurysms, using computational fluid dynamic this study has highlighted that the single LVIS device does more flow reduction than double Enterprise device but not as compared to pipeline device but double LVIS stent has better flow diverting effect than pipeline [37].

Hence after reviewing several articles related to LVIS stent in the coil embolization of intracranial aneurysms of different type and morphology this stent proved its safety, effectiveness and feasibility with its advance features and could be better option than other available stent. However, to reduce chances procedure related complication (thromboembolic and ischemic events), technical complication (rupture of aneurysms while coiling) precise knowledge about characteristic of aneurysm, anatomy of aneurysm originating artery and better 
understanding of properties of stent are required. Last but not the least further comparison study of LVIS stent vs other stent with higher population and longer follow-up is required to verify its advantages on the endovascular coiling treatment intracranial aneurysms.

\section{Conflicts of Interest}

The authors declare no conflicts of interest regarding the publication of this paper.

\section{References}

[1] Seibert, B., Tummala, R.P., Chow, R., Faridar, A., Mousavi, S.A. and Divani, A.A. (2011) Intracranial Aneurysms: Review of Current Treatment Options and Outcomes. Frontiers in Neurology, 2, 45. https://doi.org/10.3389/fneur.2011.00045

[2] Bonneville, F., Sourour, N. and Biondi, A. (2006) Intracranial Aneurysms: An Overview. Neuroimaging Clinics of North America, 16, 371-382. https://doi.org/10.1016/j.nic.2006.05.001

[3] Anson, J.A., Lawton, M.T. and Spetzler, R.F. (1996) Characteristics and Surgical Treatment of Dolichoectatic and Fusiform Aneurysms. Journal of Neurosurgery, 84, 185-193. https://doi.org/10.3171/jns.1996.84.2.0185

[4] Brown Jr., R.D. and Broderick, J.P. (2014) Unruptured Intracranial Aneurysms: Epidemiology, Natural History, Management Options, and Familial Screening. The Lancet Neurology, 13, 393-404. https://doi.org/10.1016/S1474-4422(14)70015-8

[5] Kassell, N.F., Torner, J.C., Haley Jr., E.C., Jane, J.A., Adams, H.P. and Kongable, G.L. (1990) The International Cooperative Study on the Timing of Aneurysm Surgery. Part 1: Overall Management Results. Journal of Neurosurgery, 73, 18-36. https://doi.org/10.3171/jns.1990.73.1.0018

[6] Vlak, M.H., Algra, A., Brandenburg, R. and Rinkel, G.J. (2011) Prevalence of Unruptured Intracranial Aneurysms, with Emphasis on Sex, Age, Comorbidity, Country, and Time Period: A Systematic Review and Meta-Analysis. The Lancet Neurology, 10, 626-636. https://doi.org/10.1016/S1474-4422(11)70109-0

[7] Schievink, W.I. (1997) Intracranial Aneurysms. The New England Journal of Medicine, 336, 28-40. https://doi.org/10.1056/NEJM199701023360106

[8] Nieuwkamp, D.J., Setz, L.E., Algra, A., Linn, FH., de Rooij, N.K. and Rinkel, G.J. (2009) Changes in Case Fatality of Aneurysmal Subarachnoid Haemorrhage over Time, According to Age, Sex, and Region: A Meta-Analysis. The Lancet Neurology, 8, 635-642. https://doi.org/10.1016/S1474-4422(09)70126-7

[9] Williams, L.N. and Brown Jr., R.D. (2013) Management of Unruptured Intracranial Aneurysms. Neurology Clinical Practice, 3, 99-108. https://doi.org/10.1212/CPJ.0b013e31828d9f6b

[10] Guglielmi, G. (2007) History of Endovascular Endosaccular Occlusion of Brain Aneurysms: 1965-1990. Interventional Neuroradiology, 13, 217-224. https://doi.org/10.1177/159101990701300301

[11] Byrne, J.V. and Guglielmi, G. (1998) Treatment by Endosaccular Packing with the Guglielmi Detachable Coil. In: Byrne, J.V. and Guglielmi, G., Eds., Endovascular Treatment of Intracranial Aneurysms, Springer, Heidelberg, Berlin, 133-165. https://doi.org/10.1007/978-3-642-80381-9_5

[12] Leng, B., Zheng, Y., Ren, J., Xu, Q., Tian, Y. and Xu, F. (2014) Endovascular Treat- 
ment of Intracranial Aneurysms with Detachable Coils: Correlation between Aneurysm Volume, Packing, and Angiographic Recurrence. Journal of Neurointerventional Surgery, 6, 595-599. https://doi.org/10.1136/neurintsurg-2013-010920

[13] Oishi, H., Yamamoto, M., Shimizu, T., Yoshida, K. and Arai, H. (2012) Endovascular Therapy of 500 Small Asymptomatic Unruptured Intracranial Aneurysms. American Journal of Neuroradiology, 33, 958-964. https://doi.org/10.3174/ajnr.A2858

[14] Zheng, Y., Liu, Y., Leng, B., Xu, F. and Tian, Y. (2016) Periprocedural Complications Associated with Endovascular Treatment of Intracranial Aneurysms in 1764 Cases. Journal of Neurointerventional Surgery, 8, 152-157. https://doi.org/10.1136/neurintsurg-2014-011459

[15] Chalouhi, N., Starke, R.M., Koltz, M.T., Jabbour, P.M., Tjoumakaris, S.I., Dumont, A.S., et al. (2013) Stent-Assisted Coiling versus Balloon Remodeling of Wide-Neck Aneurysms: Comparison of Angiographic Outcomes. American Journal of Neuroradiology, 34, 1987-1992. https://doi.org/10.3174/ajnr.A3538

[16] Tateshima, S., Tanishita, K., Hakata, Y., Tanoue, S.Y. and Vinuela, F. (2009) Alteration of Intraaneurysmal Hemodynamics by Placement of a Self-Expandable Stent. Laboratory Investigation. Journal of Neurosurgery, 111, 22-27. https://doi.org/10.3171/2009.2.JNS081324

[17] Zhang, X., Zhong, J., Gao, H., Xu, F. and Bambakidis, N.C. (2017) Endovascular Treatment of Intracranial Aneurysms with the LVIS Device: A Systematic Review. Journal of Neurointerventional Surgery, 9, 553-557. https://doi.org/10.1136/neurintsurg-2016-012403

[18] Feng, Z., Fang, Y., Xu, Y., Hong, B., Zhao, W., Liu, J., et al. (2016) The Safety and Efficacy of Low Profile Visualized Intraluminal Support (LVIS) Stents in Assisting Coil Embolization of Intracranial Saccular Aneurysms: A Single Center Experience. Journal of Neurointerventional Surgery, 8, 1192-1196. https://doi.org/10.1136/neurintsurg-2015-012090

[19] Bartolini, B., Blanc, R., Pistocchi, S., Redjem, H. and Piotin, M. (2014) "Y" and "X" Stent-Assisted Coiling of Complex and Wide-Neck Intracranial Bifurcation Aneurysms. American Journal of Neuroradiology, 35, 2153-2158.

[20] Inoue, A., Tagawa, M., Matsumoto, S., Nishikawa, M., Kusakabe, K., Watanabe, H., et al. (2018) Utility of Bulging Technique for Endovascular Treatment of Small and Wide-Necked Aneurysms with a Low-Profile Visualized Intraluminal Support (LVIS Jr.) Device: A Case Report and Review of the Literature. Interventional Neuroradiology, 24, 125-129. https://doi.org/10.1177/1591019917743065

[21] Spiotta, A.M., Miranpuri, A., Chaudry, M.I., Turner, R.D. and Turk, A.S. (2013) Combined Balloon Stent Technique with the Scepter C Balloon and Low-Profile Visualized Intraluminal Stent for the Treatment of Intracranial Aneurysms. Journal of Neurointerventional Surgery, 5, iii79-iii82.

https://doi.org/10.1136/neurintsurg-2012-010553

[22] Nagahama, Y., Allan, L., Nakagawa, D., Zanaty, M., Starke, R.M., Chalouhi, N., et al. (2018) Dual Antiplatelet Therapy in Aneurysmal Subarachnoid Hemorrhage: Association with Reduced Risk of Clinical Vasospasm and Delayed Cerebral Ischemia. Journal of Neurosurgery, 129, 702-710. https://doi.org/10.3171/2017.5.JNS17831

[23] Wilson, J.T., Hareendran, A., Grant, M., Baird, T., Schulz, U.G., Muir, K.W., et al. (2002) Improving the Assessment of Outcomes in Stroke: Use of a Structured Interview to Assign Grades on the Modified Rankin Scale. Stroke, 33, 2243-2246. 
https://doi.org/10.1161/01.STR.0000027437.22450.BD

[24] Mascitelli, J.R., Moyle, H., Oermann, E.K., Polykarpou, M.F., Patel, A.A., Doshi, A.H., et al. (2015) An Update to the Raymond-Roy Occlusion Classification of Intracranial Aneurysms Treated with Coil Embolization. Journal of Neurointerventional Surgery, 7, 496-502. https://doi.org/10.1136/neurintsurg-2014-011258

[25] Su, W., Zhang, Y., Chen, J., Liu, J., Rajah, G. and Yang, X. (2018) 225 Intracranial Aneurysms Treated with the Low-Profile Visualized Intraluminal Support (LVIS) Stent: A Single-Center Retrospective Study. Neurological Research, 40, 445-451. https://doi.org/10.1080/01616412.2018.1457608

[26] Fiorella, D., Arthur, A., Boulos, A., Diaz, O., Jabbour, P., Pride, L., et al. (2016) Final Results of the US Humanitarian Device Exemption Study of the Low-Profile Visualized Intraluminal Support (LVIS) Device. Journal of Neurointerventional Surgery, 8, 894-897. https://doi.org/10.1136/neurintsurg-2015-011937

[27] Cho, Y.D., Sohn, C.H., Kang, H.S., Kim, J.E., Cho, W.S., Hwang, G., et al. (2014) Coil Embolization of Intracranial Saccular Aneurysms Using the Low-Profile Visualized Intraluminal Support (LVIS) Device. Neuroradiology, 56, 543-551. https://doi.org/10.1007/s00234-014-1363-x

[28] Gross, B.A., Ares, W.J., Ducruet, A.F., Jadhav, A.P., Jovin, T.G. and Jankowitz, B.T. (2019) A Clinical Comparison of Atlas and LVIS Jr Stent-Assisted Aneurysm Coiling. Journal of Neurointerventional Surgery, 11, 171-174. https://doi.org/10.1136/neurintsurg-2018-014208

[29] Ge, H., Lv, X., Yang, X., He, H., Jin, H. and Li, Y. (2016) LVIS Stent versus Enterprise Stent for the Treatment of Unruptured Intracranial Aneurysms. World Neurosurgery, 91, 365-370. https://doi.org/10.1016/j.wneu.2016.04.057

[30] Park, S.Y., Oh, J.S., Oh, H.J., Yoon, S.M. and Bae, H.G. (2017) Safety and Efficacy of Low-Profile, Self-Expandable Stents for Treatment of Intracranial Aneurysms: Initial and Midterm Results-A Systematic Review and Meta-Analysis. Interventional Neurology, 6, 170-182. https://doi.org/10.1159/000471890

[31] Higashida, R.T., Smith, W., Gress, D., Urwin, R., Dowd, C.F., Balousek, P.A., et al. (1997) Intravascular Stent and Endovascular Coil Placement for a Ruptured Fusiform Aneurysm of the Basilar Artery. Case Report and Review of the Literature. Journal of Neurosurgery, 87, 944-949. https://doi.org/10.3171/jns.1997.87.6.0944

[32] Samaniego, E.A., Abdo, G., Hanel, R.A., Lima, A., Ortega-Gutierrez, S. and Dabus, G. (2016) Endovascular Treatment of PICA Aneurysms with a Low-Profile Visualized Intraluminal Support (LVIS Jr) Device. Journal of Neurointerventional Surgery, 8, 1030-1033. https://doi.org/10.1136/neurintsurg-2015-012070

[33] Feng, Z., Li, Q., Zhao, R., Zhang, P., Chen, L., Xu, Y., et al. (2015) Endovascular Treatment of Middle Cerebral Artery Aneurysm with the LVIS Junior Stent. Journal of Stroke and Cerebrovascular Diseases, 24, 1357-1362. https://doi.org/10.1016/j.jstrokecerebrovasdis.2015.02.016

[34] Gupta, M., Cheung, V.J., Abraham, P., Wali, A.R., Santiago-Dieppa, D.R., Gabel, B.C., et al. (2017) Low-Profile Visualized Intraluminal Support Junior Device for the Treatment of Intracranial Aneurysms. Cureus, 9, e1037.

[35] Liu, J., Zhang, Y., Wang, Y., Mu, S. and Yang, X. (2017) Stenting after Coiling Using a Single Microcatheter for Treatment of Ruptured Intracranial Fusiform Aneurysms with Parent Arteries Less than $1.5 \mathrm{~mm}$ in Diameter. World Neurosurgery, 99, 809.e7-809.e10. https://doi.org/10.1016/j.wneu.2016.12.135

[36] Poncyljusz, W., Bilinski, P., Safranow, K., Baron, J., Zbroszczyk, M., Jaworski, M., et al. (2015) The LVIS/LVIS Jr. Stents in the Treatment of Wide-Neck Intracranial 
Aneurysms: Multicentre Registry. Journal of Neurointerventional Surgery, 7, 524-529. https://doi.org/10.1136/neurintsurg-2014-011229

[37] Wang, C., Tian, Z., Liu, J., Jing, L., Paliwal, N., Wang, S., et al. (2016) Flow Diverter Effect of LVIS Stent on Cerebral Aneurysm Hemodynamics: A Comparison with Enterprise Stents and the Pipeline Device. Journal of Translational Medicine, 14, 199. https://doi.org/10.1186/s12967-016-0959-9 